\title{
Privileged heterocyclic scaffolds in chemical biology and drug discovery: synthesis and bioactivity
}

The impressive advances in the ability of organic and medicinal chemists to efficiently prepare libraries containing thousands of small organic molecules has allowed, in the last two decades, to generate molecular tools with proven utility in understanding complex biological processes and unraveling molecular dysfunctions underlying diseases, ultimately contributing to improve strategies and tools in drug discovery processes. Nevertheless, high-throughput screening of molecular libraries, through biochemical assays, often results disappointing due to the poor specificity and utility of the compounds discovered, with a negative impact on costs, in terms of time and resources employed.

Some years ago, M. E. Welsch et al. (Curr. Opin. Chem. Biol. 2010, 14, 347), examining literature reports, pointed out some limitations inherent to commercial compound libraries and collections based on bioactive natural products. Commercial compound libraries, which are produced with a focus on the overall quantity rather than on quality, suffer from limited hit rates because of the low structural diversity and poor physicochemical properties of the members in the collection (sometimes combined with the presence of undesired reactive groups). Libraries of natural products overcome issues related to hit rate, given that the parent structure has evolved over millennia for a specific pharmacological purpose, but often the collection members are simple analogs of the parent compound not conceived to explore distinct activities.

In contrast, chemical collections based on "privileged scaffolds" which are molecular frameworks of ligands in chemical libraries for an array of diverse targets (e.g., receptors), as first coined by B. E. Evans in the late 1980s (J. Med. Chem. 1988, 31, 2235), aim at overcoming some of the above limitations. The original paper of B. E. Evans referred to the benzodiazepine nucleus, as a $\beta$-turn mimetic, but bioorganic and medicinal chemistry work over the past decades has expanded the access to many privileged scaffolds, most of which are heterocyclic in nature. The progress in diversity-oriented synthesis, a concept pioneered by S. L. Shreiber (Angew. Chem., Int. $E d$. 2004, 43, 46), has allowed the privileged scaffoldbased design to evolve nowadays in a key tool for adding quality to lead generation platforms for drug discovery.

This thematic issue is not intended to comprehensively analyzing and systematically classifying the privileged heterocyclic scaffolds identified so far, neither to provide the state-of-the-art on the privileged scaffold approach as

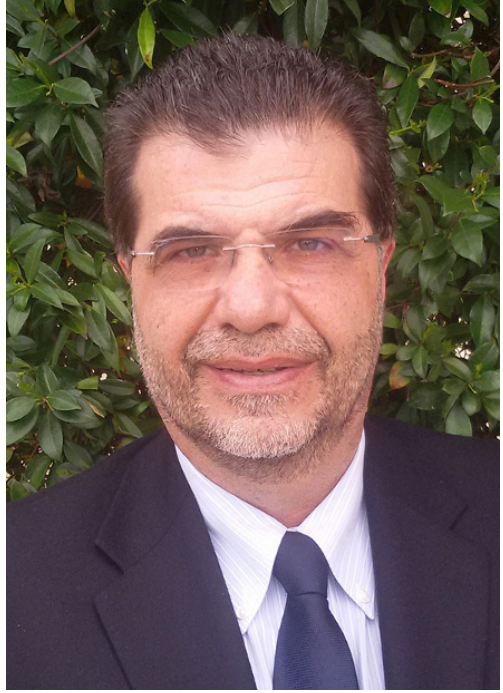

applied to library design and synthesis in drug discovery. This issue features contributions from a number of recognized scientists in the field, the purpose being to share with the readers of Chemistry of Heterocyclic Compounds scientific information about new useful approaches to the preparation of 'old' and 'new' heterocyclic scaffolds, diversity-oriented synthesis of bioactive heterocyclic derivatives and 'new' biological and pharmacological applications.

With six review articles, two microreviews (Heterocycle in Focus), nine original articles, and one short communication, authored by groups working in China, France, Italy, Latvia, The Netherlands, Norway, Russia, Spain, and Ukraine, this special issue encompasses several aspects of chemical biology and medicinal chemistry of various heterocyclic derivatives, with a focus on nature-inspired heterocyclic compounds, natural heterocyclic products and analogs, synthetic strategies and methodologies of heterocycles in drug discovery, and physicochemical profiling and structure-activity relationships of heterocyclic ligands for drug targets. On behalf of the Editorial Board, I would like to express the hope that this issue may inspire the readers in pursuing creative approaches in identifying and exploiting new privileged heterocyclic scaffolds which allow the attrition rate in drug discovery to be reduced.

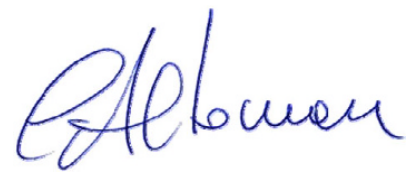

Guest Editor of the thematic issue Professor Cosimo Damiano Altomare, Department of Pharmacy - Drug Sciences, University of Bari Aldo Moro, Italy 